\title{
HGNC: the why and how of standardised gene nomenclature
}

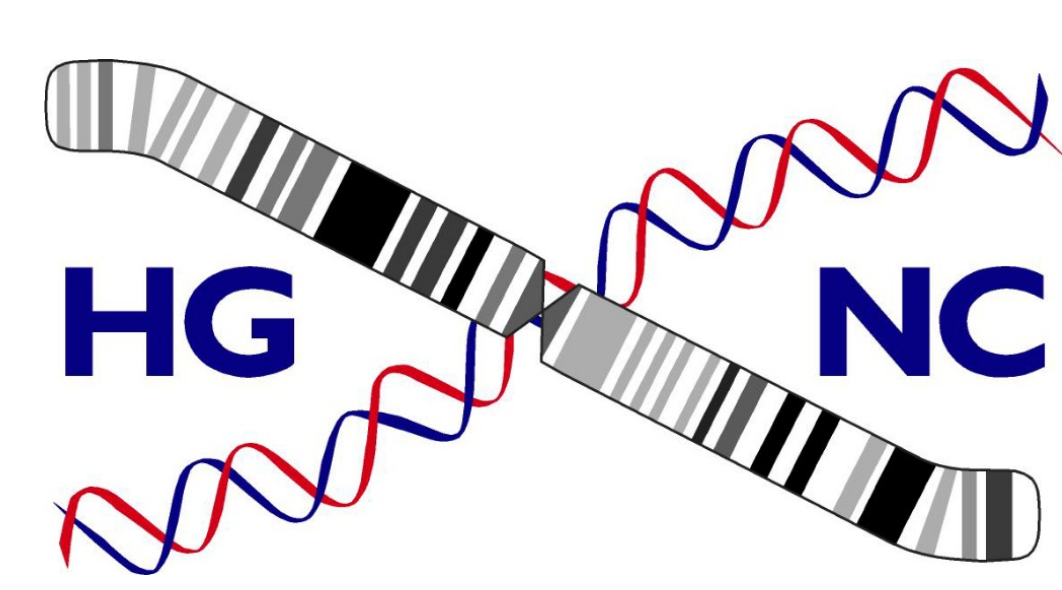

Ruth Seal, Susan Gordon, Michael Lush, Matt Wright, Elspeth Bruford

HUGO Gene Nomenclature Committee (HGNC)

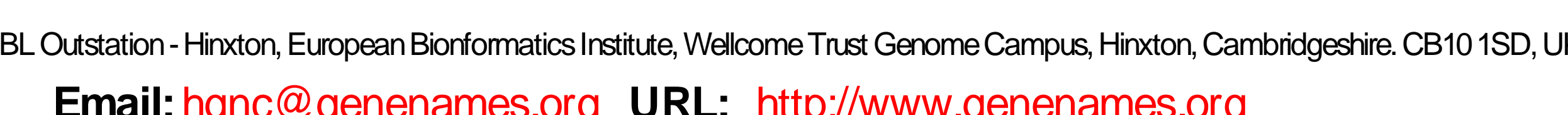
The work of the HGNC is supported by the NHGRI and the Wellcome Trust
EMBL-EBI

\section{Introduction}

Established in 1989, the HUGO Gene Nomenclature Committee (HGNC) is the only organisation authorised to assign approved nomenclature to human genes. Standardisation of gene symbols is necessary to allow researchers and curators to refer to the same gene without ambiguity and to find all relevant information for a particular gene. We currently have 28,000+ approved gene symbols in our database

(http://www.genenames.org). The majority of these symbols represent protein coding genes, however these also include pseudogenes, RNA genes, genomic features and phenotypes.

\section{Gene Symbol Assignment can be based on:}

\section{Function}

enzyme, complex subunit, receptor, transporter, binding protein ADA adenosine deaminase

TRAV16 T cell receptor alpha variable 16

GTF2H2C general transcription factor IIH, polypeptide 2C

\section{Sequence comparison}

gene family member, homolog, ' like' gene, pseudogene

MAGEA5 melanoma antigen family A, 5

MIOS missing oocyte, meiosis regulator, homolog (Drosophila)

NF1L7 neurofibromin 1-like 7

NCLP1 nucleolin pseudogene 1

\section{Domain structures}

intrinsic domains and motifs

AKD1 adenylate kinase domain containing 1

TRIM32 tripartite motif-containing 32

Other relevant information from researchers and databases

associated phenotype, cellular location, chromosomal location

Examples:

DFNB17 deafness, autosomal recessive 17

ERP27 endoplasmic reticulum protein 27

C11orf21 chromosome 11 open reading frame 21

All gene symbols must be UNIQUE and should ideally have all of the following properties:

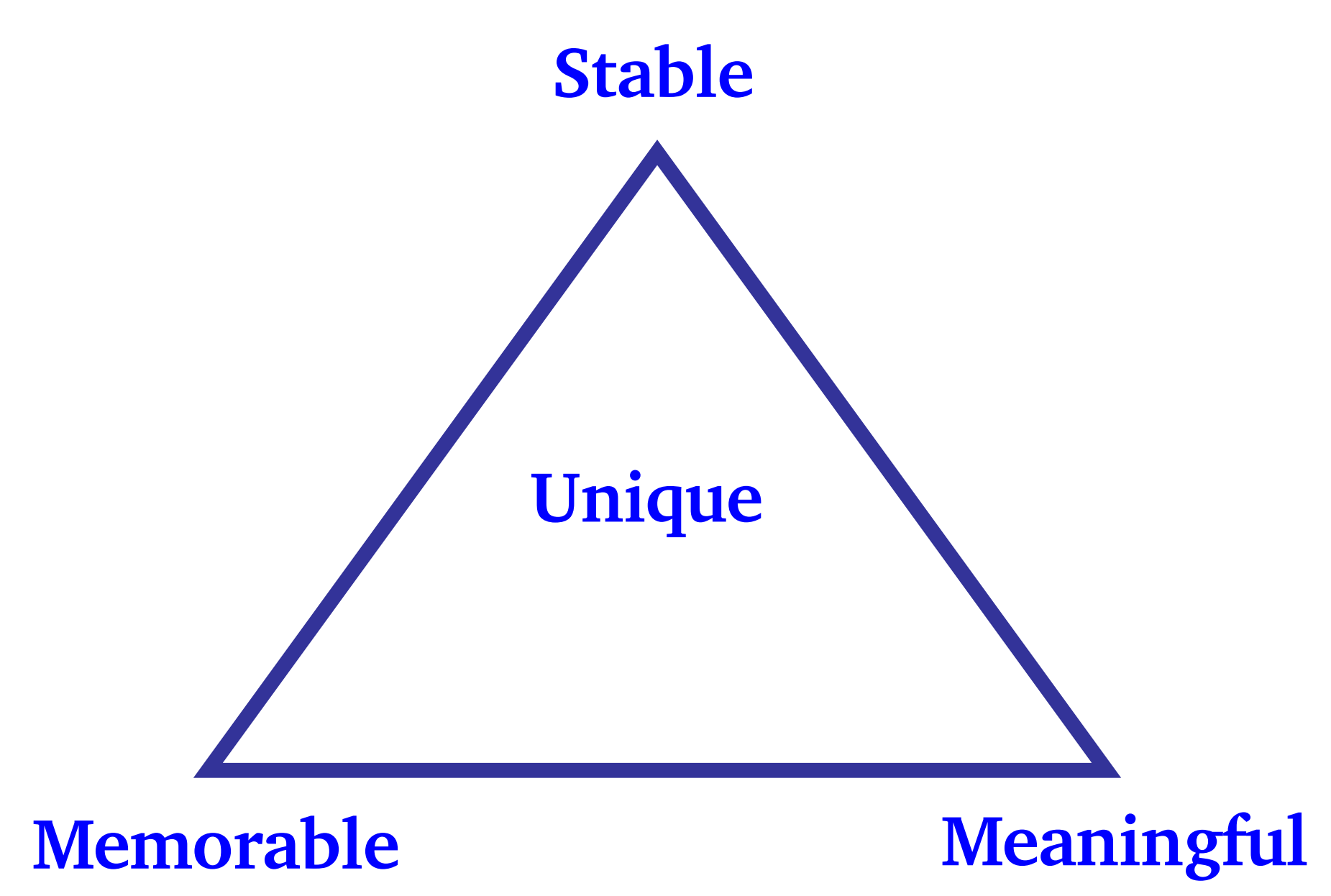

In reality, deciding on a suitable gene symbol often requires compromise.

Here are examples of scenarios that regularly arise:

Scenario \#1: The published or proposed symbol or its root symbol is not unique.

Published symbol: Der1 " derlin-1" (in mouse)

Stem symbol " Der" is already in use for " delayed early response" genes in mouse Action: coordinated with the MGNC to approve unique symbols

Conclusion: approved unique symbol $D E R L 1$

Der1-like domain family, member 1"

8

Scenario \#2: The published or proposed symbol matches a word or abbreviation that would cause problems with database searching.

Published symbol: RAIN “ RAs-INteracting protein"

Number of Pubmed hits to RAIN: over 7500

Action: Contacted authors to negotiate more appropriate symbol

Conclusion: Approved author-agreed symbol RASIP1

Ras interacting protein 1"

$\&^{2} s$

Scenario \#3: A single gene has been published using numerous symbols

Published symbols: MAVS, VISA, IPS-1, Cardif

Action: as only one of these symbols, MAVS, does not contravene our guidelines,

the authors were contacted to promote use of this symbol

Conclusion: approved unique symbol MAVS

mitochondrial antiviral signaling protein"
CASP8: A gene symbol in HGNC and beyond
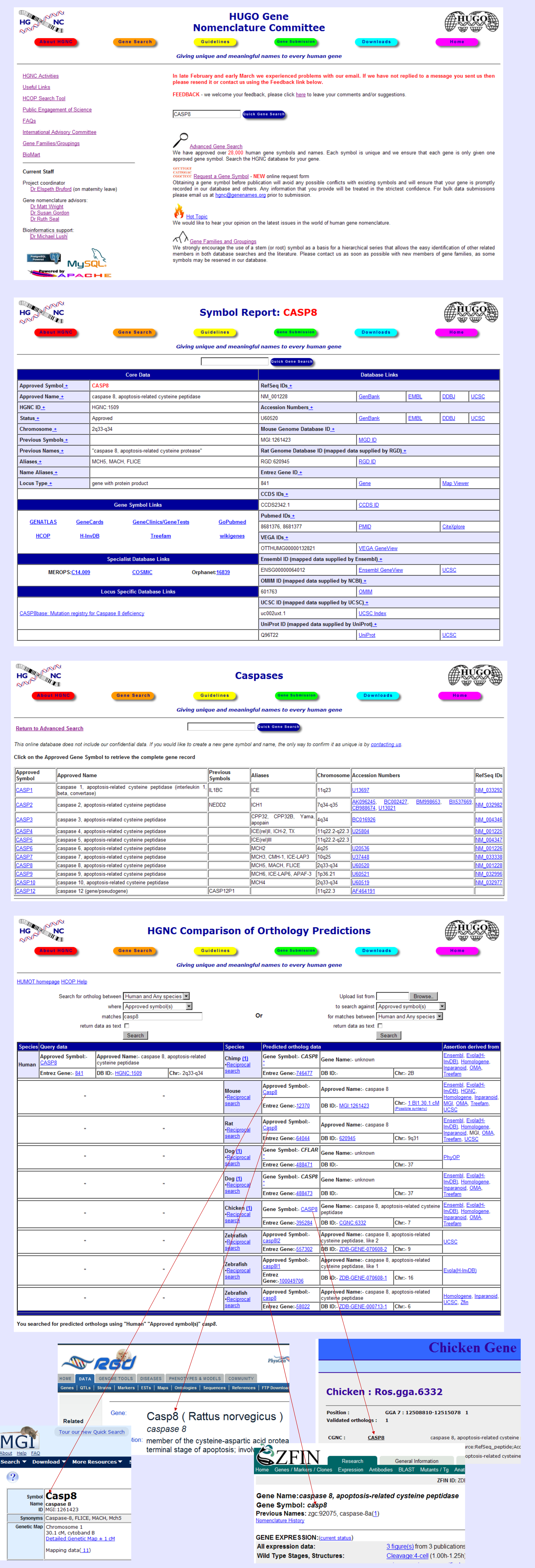